\section{References and Notes}

(1) Droske, J. P.; Stille, J. K.; Alston, W. B. Macromolecules 1984, $17,14$.

(2) Sutherlin, D. M.; Stille, J. K. Macromolecules 1986, 19, 251.

(3) Sutherlin, D. M.; Stille, J. K.; Alston, W. B. Macromolecules $1986,19,257$.

(4) (a) Sivaramakrishnan, K. P., Samyn, C.; Westerman, I. J.; Wong, D. T.-M. Marvel, C. S. J. Polym. Sci., Polym. Chem. Ed. 1975, 13, 1083. (b) Chang, D. M.; Marvel, C. S. J. Polym. Sci., Polym. Chem. Ed. 1975, 13, 2507.

(5) Meyers, R. A.; Hamersma, J. W.; Green, H. E. Polym. Lett. $1972,10,685$.

(6) Lin, S.; Marvel, C. S. J. Polym. Sci., Polym. Chem. Ed. 1983, 21,1151 .
(7) Chow, S. W.; Loeb, W. E.; White, C. E. J. Appl. Polym. Sci. $1969,13,2325$.

(8) Chow, S. W.; Pilato, L. A.; Wheelwright, W. L. J. Org. Chem. $1970,35,20$

(9) Droske, J. P.; Stille, J. K. Macromolecules 1984, 17, 1.

(10) Norris, S. O.; Stille, J. K. Macromolecules 1976, 9, 496.

(11) Jones, M. E. B.; Thornton, D. A.; Webb, R. F. Makromol. Chem. 1961, 49, 62 .

(12) Beever, W. H.; Stille, J. K. J. Polym. Sci., Polym. Symp. 1978, 65,41 .

(13) (a) Cram, D. J.; Fischer, H. P. J. Org. Chem. 1965, 30, 1815. (b) Cram, D. J.; Allinger, N. L. J. Am. Chem. Soc. 1955, 77, 6289.

\title{
Rheology of a Twin Liquid Crystalline Polymer
}

\author{
Y. G. Lin, R. Zhou, J. C. W. Chien, and H. H. Winter* \\ Chemical Engineering Department and Polymer Science and Engineering Department, \\ University of Massachusetts, Amherst, Massachusetts 01003. Received December 22, 1987
}

\begin{abstract}
Phase transitions of a twin liquid crystalline polymer (TLCP), which was synthesized from 4-[(4'-alkoxybenzoyl)oxy]benzoyl chloride and $\alpha, \omega$-dihydroxy-telechelic polytetrahydrofuran, have been determined by measurements of linear viscoelastic properties. In the phase-separated mesophase state, the TLCP displayed high elasticity to small-amplitude deformation, due to mesogen-induced physical cross-linking. As the strain amplitude increased, shear thinning affected both dynamic and steady flow properties. This effect is assumed to be due to a reduction of tie molecules and an increase of loops in the physical network. If the average shear rate of oscillatory flow was defined as $\bar{\gamma}_{d}=4 \gamma_{0} \omega / 2 \pi$, a good agreement of $\eta^{*}\left(\bar{\gamma}_{d}\right) \approx \eta(\dot{\gamma})$ was found in the shear thinning region and the high shear rate plateau. After shear thinning a TLCP, structural recovery took several days, unless the sample was temporarily heated to the isotropic state. However, above the clearing temperature of about $65^{\circ} \mathrm{C}$, the polymer became single phased and showed rheological behavior typical for a polymeric melt of low molecular weight.
\end{abstract}

\section{Introduction}

Polymers in the liquid crystalline state form anisotropic textures due to the interaction of the mesogenic groups. ${ }^{1-8}$ As a result, the basic flow units are not only molecular chains, but also macrostructural units in which a large number of molecular chains move cooperatively. This gives rise to unusual rheological phenomena, including long relaxation time, ${ }^{3,9-12}$ elasticity,, $32-14$ and distinct shear thinning effect at low shear rates. ${ }^{1-6,11,14,15}$ It is apparent that the study of the rheology of liquid crystalline polymers is still in its infancy and much more research in a wide variety of liquid crystalline polymers is needed to improve our understanding in this field.

A twin liquid crystalline polymer (TLCP) ${ }^{16}$ consists of a flexible center chain with mesogenic segments on both ends. Below the clearing temperature (about $65^{\circ} \mathrm{C}$ for this TLCP), mesogenic ends form a separated nematic phase. In the present work, rheology of a TLCP was studied in both the microphase-separated state and the compatible isotropic state, using dynamic as well as steady flow measurements. The experimental observations are related to physical cross-linking through phase-separated mesogen domains.

\section{Experimental Section}

Materials. The TLCP studied in this work was synthesized from 4-[(4'-alkoxybenzoyl)oxy]benzoyl chloride ${ }^{16}$ and $\alpha, \omega$-dihydroxy-telechelic polytetrahydrofuran of $2000 M_{\mathrm{w}}$, having the following structure as determined by NMR:

$$
\mathrm{CB}_{3} \mathrm{CB}_{2} \mathrm{CB}_{2} \mathrm{CB}
$$

The DSC thermogram of the polymer displayed a large endothermic peak at $24^{\circ} \mathrm{C}$ and a very small one at $55^{\circ} \mathrm{C}$, corresponding to the phase transitions of crystal to liquid crystal and liquid crystal to isotropic, respectively. The enthalpies and entropies of these transitions have been reported. ${ }^{16}$ Under the polarizing microscope, dispersed liquid crystalline domains were observed in the temperature range $24-55^{\circ} \mathrm{C}$, but they completely disappeared above $65^{\circ} \mathrm{C}$

Apparatus. Dynamic and steady shear flow measurements were performed on a Rheometrics dynamic spectrometer (RDS) and a Rheometrics stress rheometer (RSR), respectively, using the cone/plate geometry. Samples were molded in the rheometers at $75^{\circ} \mathrm{C}$. On RDS, in addition to linear viscoelastic properties, we measured the apparent storage modulus $G_{a}^{\prime}$, loss modulus $G_{a}^{\prime \prime}$, and complex viscosity $\eta_{\mathrm{a}}^{*}$ at large strain amplitude beyond the linear viscoelastic region.

Phase Transitions. Figure la shows the storage modulus $G^{\prime}$ and the loss modulus $G^{\prime \prime}$ of the TLCP, measured at a frequency of $10 \mathrm{rad} / \mathrm{s}$ and in the linear viscoelastic region, as a function of temperature. From 20 to $32{ }^{\circ} \mathrm{C}$, the moduli $G^{\prime}$ and $G^{\prime \prime}$ decayed by 2 orders of magnitude, due to the transition of the crystalline state to a mesophase state. In the mesophase state, $G^{\prime}$ was still higher than $G^{\prime \prime}$, and both moduli decreased gradually with increasing temperature. The transition from the mesophase state to the isotropic state started at $45^{\circ} \mathrm{C}$ and completed at about $65^{\circ} \mathrm{C}$, accompanied by $10^{3}$ - and $10^{2}$-fold decrease in $G^{\prime}$ and $G^{\prime \prime}$, respectively. These phase-transition temperatures as measured with linear viscoelastic properties are in good agreement with those determined by DSC and polarizing microscopy. ${ }^{16}$ By comparison, $\alpha, \omega$-dihydroxy-telechelic polytetrahydrofuran did not display a mesophase state by itself. Above the melting temperature at 30 ${ }^{\circ} \mathrm{C}$, both moduli $G^{\prime}$ and $G^{\prime \prime}$ decreased regularly as the temperature increased (Figure 1b).

In the present work, the rheology of this TLCP is characterized at $45{ }^{\circ} \mathrm{C}$ (liquid crystalline state), $55^{\circ} \mathrm{C}$ (middle point of the transition zone), and $65^{\circ} \mathrm{C}$ (isotropic state).

Linear viscoelastic properties of the TLCP at the three temperatures are shown in Figure 2. At $45^{\circ} \mathrm{C}$, the polymer melt exhibited a high storage modulus $G^{\prime}$, almost like an elastomer. At $65^{\circ} \mathrm{C}, G^{\prime}$ was much lower than $G^{\prime \prime}$ in the experimental frequency range, i.e., the polymer melt was essentially characterized 


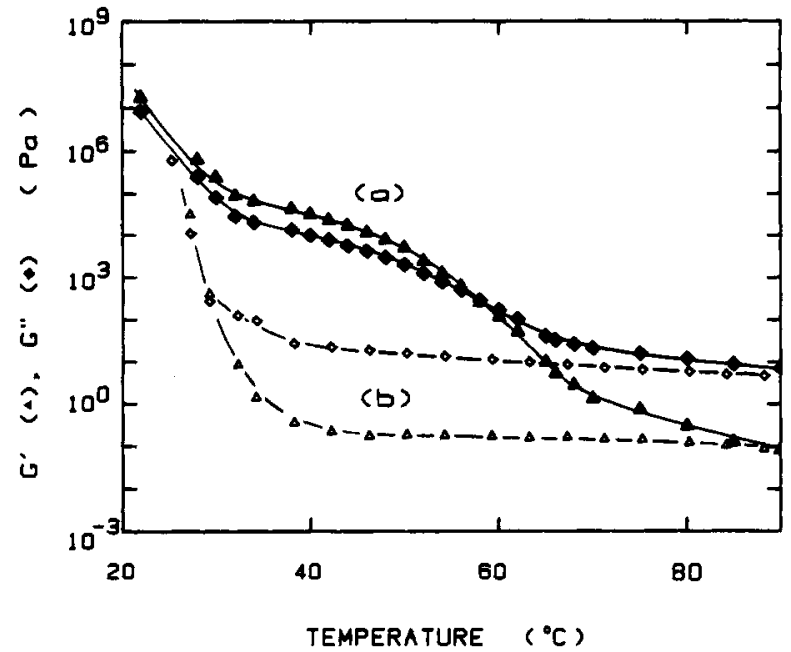

Figure 1. Temperature sweep at a frequency of $\omega=10 \mathrm{rad} / \mathrm{s}$. The strain amplitude, $\gamma_{0}<0.3$, was in the linear viscoelastic region: (a) TLCP; (b) PTHF with molecular weight $M_{\mathrm{w}}=2000$.
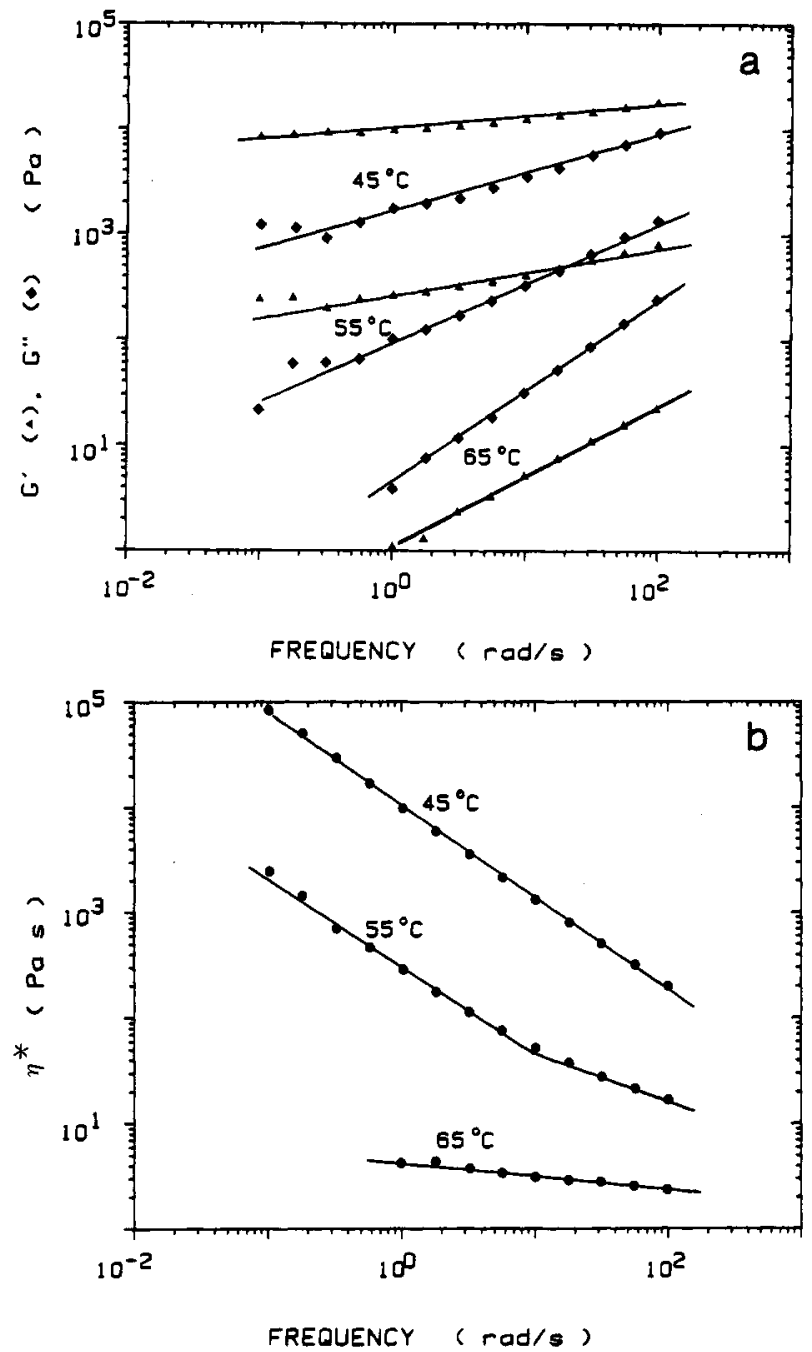

Figure 2. Frequency sweep at the three temperatures.

by its viscosity, as is typical for low molecular weight polymers. The data can be replotted in the form of the dynamic viscosity $\eta^{*}=\left(G^{\prime 2}+G^{\prime \prime 2}\right)^{0.6} / \omega$, see Figure $2 b$. The strong frequency dependence at $45^{\circ} \mathrm{C}\left(\mathrm{d} \log \eta^{*} / \mathrm{d} \log \omega=0.9\right)$ disappears upon heating to $65^{\circ} \mathrm{C}\left(\mathrm{d} \log \eta^{*} / \mathrm{d} \log \omega=-0.2\right)$. At an intermediate temperature $\left(55^{\circ} \mathrm{C}\right)$, however, the polymer melt behaved distinctly different, showing two power law regions.

Effect of oscillatory shear amplitude on the dynamic mechanical properties is displayed in Figure 3 . At $45^{\circ} \mathrm{C}$, the polymer

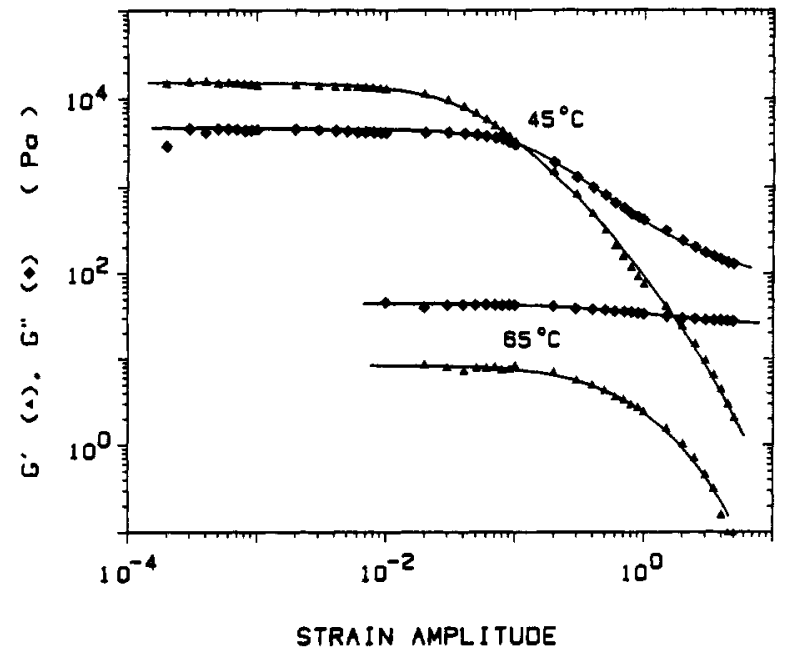

Figure 3. Strain sweep at a frequency of $\omega=10 \mathrm{rad} / \mathrm{s}$.
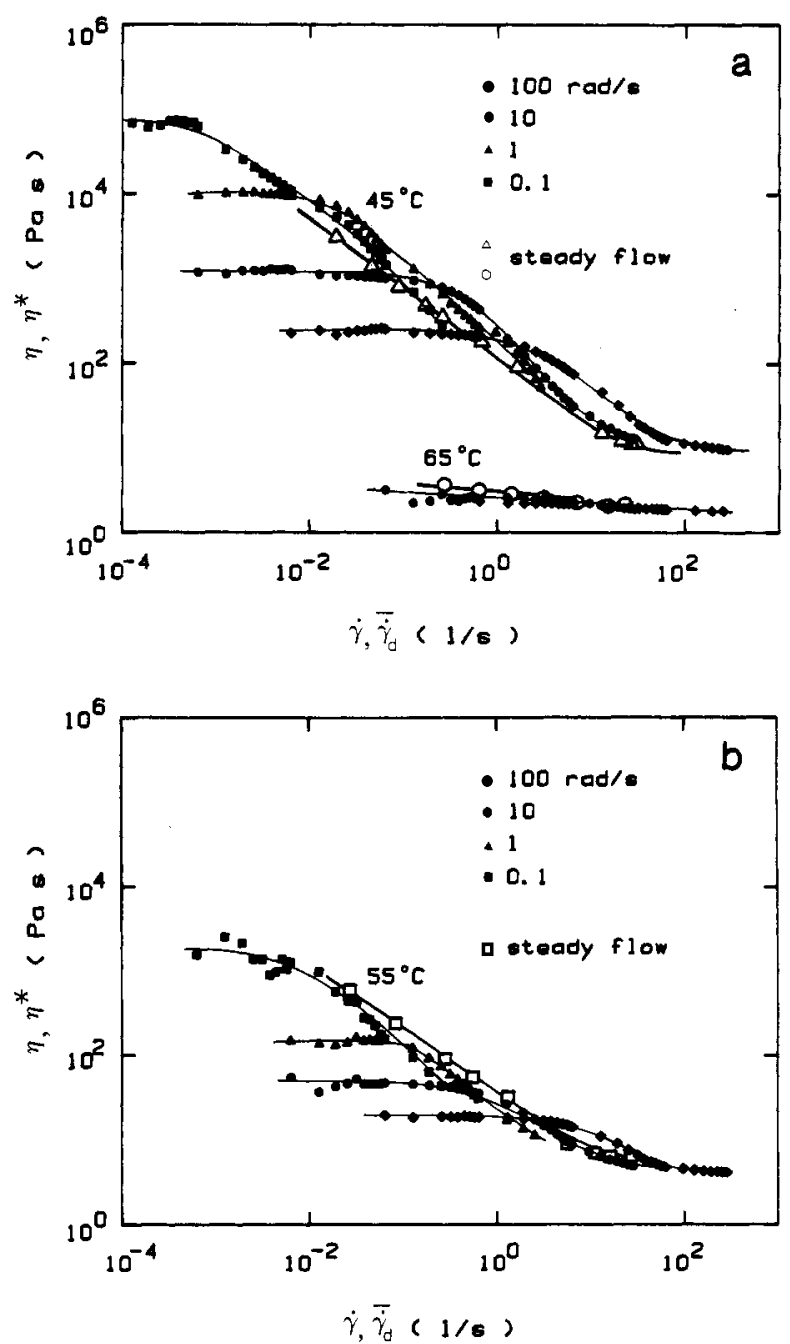

Figure 4. Shear thinning in steady and dynamic shear flows. For the dynamic flow, the average shear rate is defined as $\bar{\gamma}_{d}=$ $4 \gamma_{0} \omega / 2 \pi$.

showed high elasticity in the linear viscoelastic region $\left(\gamma_{0}<10^{-2}\right)$. As the strain amplitude increased beyond the linear viscoelastic region, both $G^{\prime}$ and $G^{\prime \prime}$ decayed by several orders of magnitude. The storage modulus decreased much more than the loss modulus, indicating that shearing at large strain amplitude transforms the polymer into a viscous liquid. By comparison, at $65^{\circ} \mathrm{C}$, the viscous behavior dominated in the entire range.

Effect of Shear Rate on Viscosity. On the Rheometrics stress rheometer (RSR), samples were sheared under constant stress. The resulting shear rate was measured after having reached 

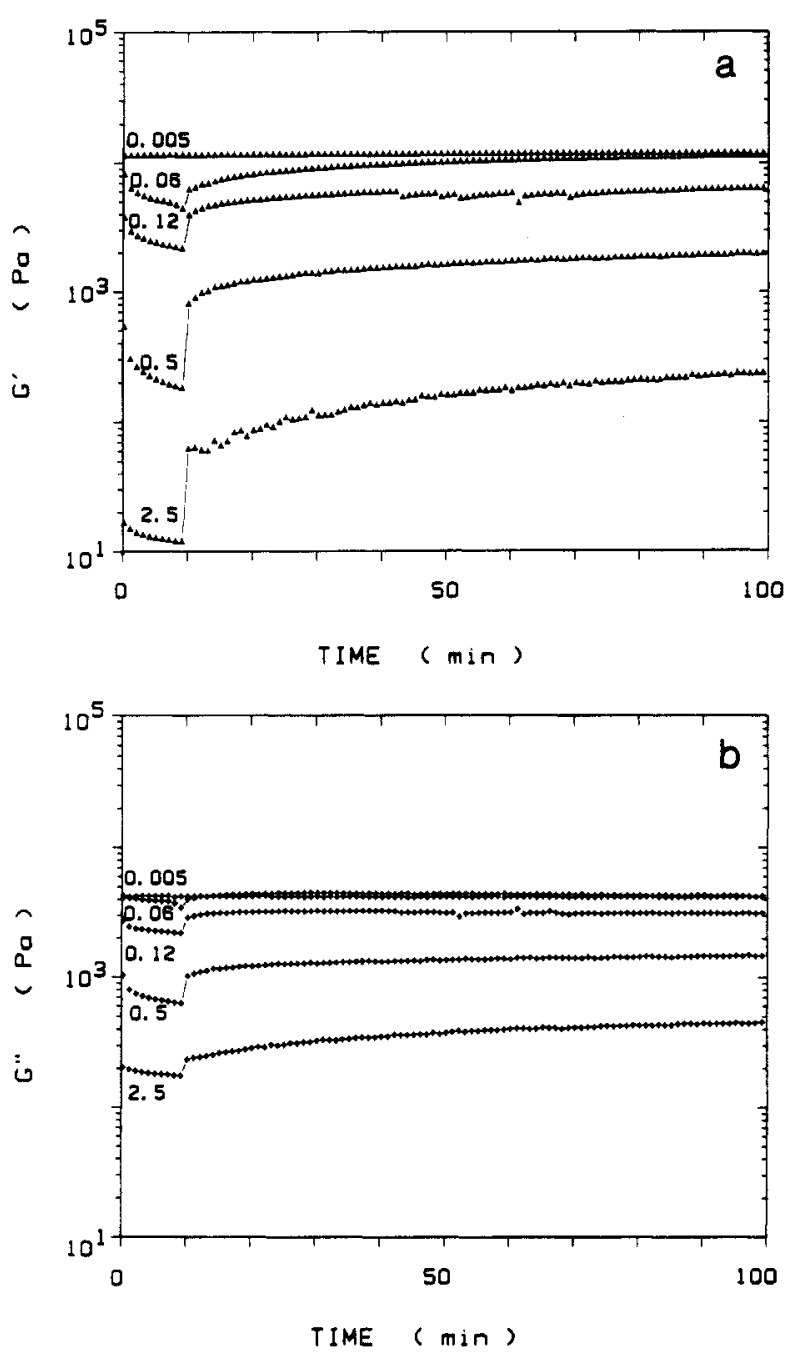

Figure 5. Time sweep after $10 \mathrm{~min}$ of dynamic shearing at various strain amplitudes: $T=45^{\circ} \mathrm{C}, \omega=10 \mathrm{rad} / \mathrm{s}, \gamma_{0}=0.005$.

steady flow. In Figure 4, the viscosity $\eta$ is plotted as a function of shear rate $\dot{\gamma}$. Shear thinning was observed at 45 and $55^{\circ} \mathrm{C}$, the viscosity having a slope $(\mathrm{d} \log \eta / \mathrm{d} \log \gamma$ ) of -0.82 and -0.72 , respectively. Beyond this shear thinning region, the viscosity leveled off to a lower plateau value. Note that in the shear thinning region, $\eta(\dot{\gamma})$ was $1-2$ orders of magnitude lower than corresponding value of $\eta^{*}(\omega)$ (compare to Figure 2), indicating that the TLCP does not follow the Cox-Merz rule.

For comparing oscillatory to steady shear data, we replace the periodic shear rate

$$
\dot{\gamma}_{d}=\gamma_{0} \omega \cos (\omega t)
$$

with an average shear rate as

$$
\dot{\bar{\gamma}}_{\mathrm{d}}=\omega / 2 \pi \int_{0}^{2 \pi / \omega}\left|\dot{\gamma}_{\mathrm{d}}\right| \mathrm{d} t=4 \gamma_{0} \omega / 2 \pi
$$

In Figure $4, \eta_{\mathrm{a}}^{*}$ is plotted as a function of $\bar{\gamma}_{\mathrm{d}}$. It can be seen that at 45 and $55{ }^{\circ} \mathrm{C}$, the apparent dynamic viscosity $\eta_{\mathrm{a}}^{*}\left(\bar{\gamma}_{\mathrm{d}}\right)$ had three distinct regions: (i) linear viscoelastic region $\left(\gamma_{0}<10^{-2}\right)$, in which $\eta^{*}$ was essentially determined by the frequency $\omega$, less dependent on the $\bar{\gamma}_{d}$; (ii) shear thinning region $\left(\gamma_{0}>10^{-2}, \bar{\gamma}_{d}<\right.$ 50) where $\eta^{*}{ }_{a}$ decreased as a function of $\bar{\gamma}_{d}$; (iii) lower plateau at high shear rates $\left(\bar{\gamma}_{\mathrm{d}}>50\right)$, having nearly constant $\eta_{\mathrm{a}}^{*}$. Worthy of note is the good agreement of $\eta(\dot{\gamma})$ with $\eta^{*}{ }_{a}\left(\bar{\gamma}_{d}\right)$ in the shear thinning region and in the lower plateau at high shear rates.

At $65^{\circ} \mathrm{C}$, the polymer did not display significant shear thinning at the experimental shear rates. Oscillatory and steady shear data gave the same viscosity.

Recovery of modulus after large amplitude shear was studied on the RDS rheometer at $45^{\circ} \mathrm{C}$ (Figure 5). Samples were first dynamically sheared with various strain amplitudes $\left(\gamma_{0}=\right.$

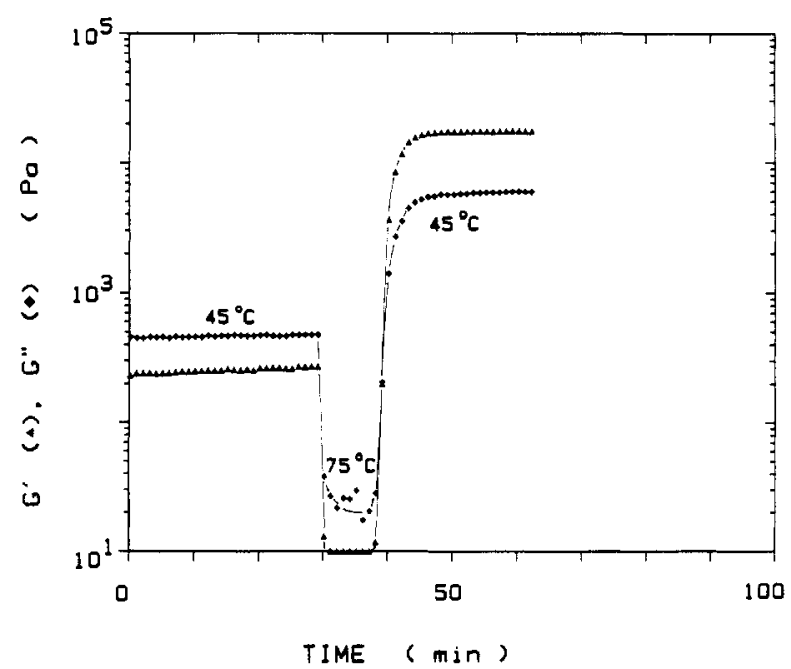

Figure 6. Structural recovery by a heating to the isotropic state. Before the measurement $\left(\omega=10 \mathrm{rad} / \mathrm{s}, \gamma_{0}=0.005\right)$, the sample has been subjected to dynamic shearing with large strain amplitude $\left(\gamma_{0}=2.5\right)$ for $10 \mathrm{~min}$, and with a small strain amplitude $\left(\gamma_{0}=0.005\right)$ for the following $90 \mathrm{~min}$.

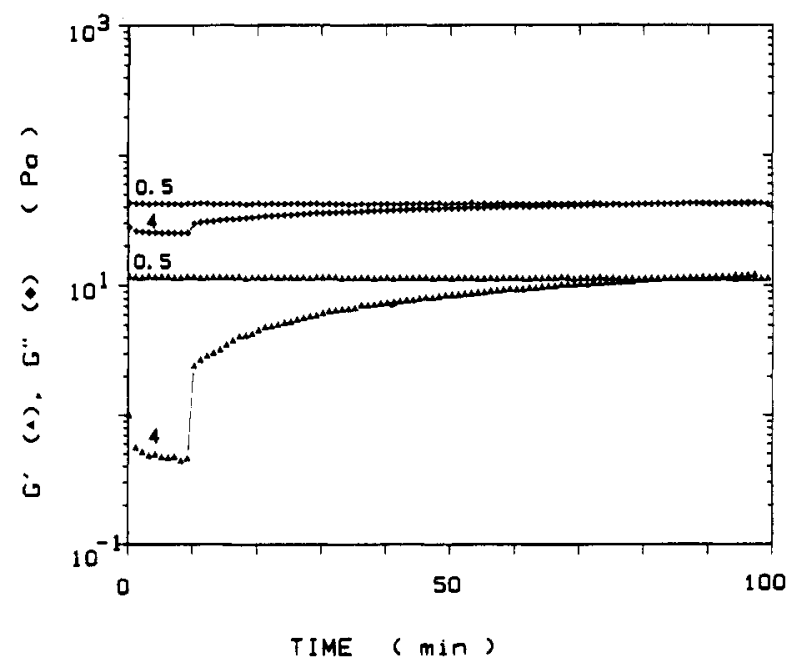

Figure 7. Time sweep with $T=65^{\circ} \mathrm{C}, \omega=10 \mathrm{rad} / \mathrm{s}$, and $\gamma_{0}=$ 0.5 , after dynamic shearing at the indicated strain amplitude for the first $10 \mathrm{~min}$.

$0.005,0.06,0.12,0.5,2.5)$ for $10 \mathrm{~min}$, and then the $G^{\prime}$ and $G^{\prime \prime}$ of the samples were measured at a small strain amplitude $\left(\gamma_{0}=0.005\right)$ for the next $90 \mathrm{~min}$. The extent of shear thinning during the large-amplitude shear depended on the applied strain amplitude. It seems that a transition from elastic dominant to viscosity dominant behavior occurred at a critical strain amplitude of $\gamma_{0}$ $=0.1$ (see also strain sweep of Figure 3). A sample sheared with a strain amplitude smaller than 0.1 (e.g., $\gamma_{0}=0.06$ ) could completely recover in $80 \mathrm{~min}$. But the samples which had been sheared at strain amplitudes larger than 0.1 (e.g., $\gamma_{0}=0.12,0.5,2.5$ ) could not recover quickly. A complete recovery, if that is possible, would take several days.

Rapid recovery can be induced by temporary heating to the isotropic state. For this experiment, a sample was sheared at a strain amplitude of $\gamma_{0}=2.5$ for $10 \mathrm{~min}$, relaxed for $90 \mathrm{~min}$ (the lowest curve in Figure 5), kept at $45^{\circ} \mathrm{C}$ for 30 more min, heated to $70^{\circ} \mathrm{C}$ for $7 \mathrm{~min}$, and then cooled back to $45^{\circ} \mathrm{C}$. During this process, $G^{\prime}$ and $G^{\prime \prime}$ were measured with a small strain amplitude of $\gamma_{0}=0.005$. Figure 6 shows that both $G^{\prime}$ and $G^{\prime \prime}$ returned to their original level as soon as the temperature was reset at $45^{\circ} \mathrm{C}$ (Figure 5).

A similar experiment was performed for the polymer melt at $65^{\circ} \mathrm{C}$. A sample was first sheared with a large strain amplitude of $\gamma_{0}=4$ for $10 \mathrm{~min}$, and then the $G^{\prime}$ and $G^{\prime \prime}$ were measured with a small strain amplitude $\left(\gamma_{0}=0.5\right)$. Initially, the large amplitude shearing decreased the moduli, but the entire decrease in modulus 
was regained in $70 \mathrm{~min}$ after switching to the small strain amplitude (Figure 7).

\section{Discussion}

Physical Cross-Linking. As shown by polarizing microscopy, ${ }^{16}$ the mesophase state of the TLCP has a two-phase domain structure with dispersed liquid crystalline domains in a continuous matrix of flexible PTHF chains. The polymer is opaque before the transition to the isotropic state. This indicates that the average linear size of the liquid crystalline domains is on the same order as or larger than the wavelength of the visible light. For the mechanical properties it is important whether a major fraction of the TLCP molecules form loops with both mesogenic ends in the same domain (reentry molecules) or whether they form connecting chains with two ends belonging to separated domains (tie molecules). In the latter case, the dispersed liquid crystalline domains act as physical cross-links, tying the flexible PTHF chains into a temporary three-dimensional network. In the linear viscoelastic region (small strain amplitude, $\gamma_{0}<10^{-2}$ ), the physical cross-linking is maintained and the polymer behaves like an elastomer, showing high elasticity and weak frequency dependence of the dynamic modulus (Figure 2).

Shear-induced structural changes might be explained by the following mechanism. Liquid crystalline domains are held together by phase separation forces and are, obviously, not as strong as chemical cross-links. While the application of large strain is expected to have little effect on the reentry molecules, it significantly affects the tie molecules: the mesogenic ends of tie molecules are pulled out of and newly associate into phase-separated liquid crystalline domains. Both reentry and tie molecules may form when the mesogenic groups associate again and, as a result of this process, the ratio of tie to reentry molecules decreases and the network degenerates. This hypothesis explains the $10^{2}-10^{4}$-fold decay of the mechanical properties and the transition from elasticitydominant to viscosity-dominant behavior (Figure 3).

As shown in Figures 3 and 4 , at $45^{\circ} \mathrm{C}$, the smallest strain amplitude to induce structural changes is on the level of $10^{-2}$, independent of frequency. When the strain exceeds this critical level, the power law of the viscosity versus shear rate (Figure 4) suggests that the breakdown of the physical cross-linking depends on the rate of shear energy density, $\tau \dot{\gamma}$.

The disappearance of tie molecules and the formation of reentry chains might also explain the existence of the low-viscosity plateau at high shear rates (Figure 4). All the tie molecules have been removed and the liquid crystalline domains are "wrapped" by a layer of reentry chains. Because the weight fraction of mesogenic ends is $23 \%$, and the volume fraction is even smaller, ${ }^{16}$ the PTHF reentry chain layer is relatively thick. This layer suppresses the interaction of liquid crystalline domains. As a result, the recovery of the network structure takes very long times once the physical cross-linking of the mesophase state is modified by larger shear, as shown by the dynamic mechanical spectroscopy (Figure 5). However, if a sheared sample is heated to the compatible isotropic state (erasing the thermomechanical history of sample) and then cooled to the mesophase state, the two-phase structure with an equilibrium ratio of tie to reentry molecules will be easily reformed (Figure 6).

Isotropic State. It should be emphasized that, for our polymer, the nematic/isotropic transition within the mesogen domains is accompanied by a dissolution of the domains themselves. After the transition, the mesogens become miscible with the center chain PTHF. The mo- lecular ends lose their ability for physical cross-linking. In fact, the polymer melt above $65^{\circ} \mathrm{C}$ is colorless and completely transparent, indicating the absence of inhomogeneous texture. The mechanical properties indicate that the molecular weight of the TLCP is far below the critical value to induce entanglements. In the isotropic state, the polymer has very low viscosity and displays the typical rheological behavior of a low molecular weight polymer.

Comparison with Main-Chain LCPs. At the onset of nematic state, the melt viscosity of a liquid crystalline copolyester could be less than that of a similar but nonmesogenic polymer by over 3 orders of magnitude. ${ }^{17}$ Usually, the clearing transition of main-chain LCPs is characterized by an increase in the shear viscosity. ${ }^{17,18}$ This seems to be in contradiction to what was observed for the TLCP. However, in our case, lower viscoelastic properties of the isotropic state can be attributed to the disappearance of the microphase-separated mesogen domains.

In the shear thinning region, the TLCP displays a power law exponent $\left(-0.82\right.$ at $45^{\circ} \mathrm{C},-0.72$ at $\left.55^{\circ} \mathrm{C}\right)$ much greater than that of main-chain LCPs, e.g., that of wholly aromatic copolyesters ranges from -0.4 to $-0.6 .^{3,14,15}$ The rheology of main-chain LCPs is largely interpreted by the domain flow mechanism, and the shear thinning is attributed to the breakdown of domains. However, for the TLCP, a liquid crystalline domain is not an independent flow unit, due to a large number of connecting chains between the domains. When the TLCP is sheared, the breakdown of liquid crystalline domains could be accelerated by the extra force transferred by the connecting chains. The resulting collapse of physical cross-linking is mirrored in the decline of the viscoelastic properties.

\section{Conclusion}

Dynamic measurement of linear viscoelastic properties is found to be a sensitive method to determine the phase transitions of the TLCP. In fact, the transition from the phase-separated mesophase state to the compatible isotropic state is clearly observed by pronounced decrease in the complex viscosity and the relative elasticity.

In the mesophase state, the mesogenic ends of the TLCP form phase-separated liquid crystalline domains and tie the flexible PTHF center chains in a three-dimensional network. This physically cross-linked structure is responsible for the relatively high elasticity at small deformation. However, since the mesogens are held together in domains by relatively weak van der Waals forces, the mesogen-induced cross-linking shows little resistance to large amplitude shearing. Compared to main-chain liquid crystalline polymers, the TLCP displays more pronounced shear thinning behavior. The shear modification is reversible. At rest, physical cross-links of the sheared TLCP recover very slowly by forming tie molecules between phase-separated mesogen domains.

Acknowledgment. This work is supported by Contract N00014-85-k-0880 of the Office of Naval Research.

Registry No. Polytetramethylene glycol bis[4-((4-butoxybenzoyl)oxy) benzoate], 110015-78-6.

\section{References and Notes}

(1) Onogi, S.; Asada, T. In Rheology and Rheo-optics of Polymer Liquid Crystals; Astariata, G., Marrucci, G., Eds.; papers presented at Eighth International Congress on Rheology, Naples, 1980; Plenum: New York, 1980; Vol. 1, p 127.

(2) Asada, T. In Polymer Liquid Crystals; Ciferri, A., Krigbaum, W. R., Meyer, R. B., Eds.; Academic: New York, 1982; Chapter 9.

(3) Wissbrun, K. F. Br. Polym. J. 1980, 12, 163.

(4) Marrucci, G. Proc. Int. Congr. Rheol., 9th 1984.

(5) Wissbrun, K. F. Faraday Discuss. Chem. Soc. 1985, 79, 161. 
(6) Graziano, D. J.; Mackley, M. R. Mol. Cryst. Liq. Cryst. 1984, $106,73$.

(7) Marrucci, G. Pure Appl. Chem. 1985, 57, 1545.

(8) Wood, B. A.; Thomas, E. L. Nature (London) 1986, 324, 655

(9) Onogi, Y.; White, J. L.; Fellers, J. F. J. Non-Newtonian Fluid Mech. 1980, 7, 121.

(10) Graziano, D. J.; Mackley, M. R. Mol. Cryst. Liq. Cryst. 1980, $106,73$.

(11) Viola, G. G.; Baird, D. G. J. Rheol. 1986, 30 (3), 601.

(12) Lin, Y. G.; Winter, H. H. Liq. Cryst., in press.
(13) Lin, Y. G.; Winter, H. H.; Lieser, G. Liq. Cryst., in press.

(14) Wissbrun, K. F.; Kiss, G.; Cogswell, F. N. Chem. Eng. Commun. 1987, 53, 149.

(15) Kiss, G. J. Rheol. 1986, 30 (3), 585.

(16) Chien, J. C. W.; Zhou, R.; Lillya, C. P. Macromolecules 1987 $20,2340$.

(17) Dobb, M. G.; McIntyre, J. E. J. Polym. Sci., Polym. Symp. $1978,63,67$.

(18) Wissbrum, K. F.; Griffin, A. C. J. Polym. Sci., Polym. Phys. Ed. 1982, 20, 1835 .

\title{
Pyrolysis-Field Ionization Mass Spectrometry of Epoxy Resins
}

\author{
Bernd Plage and Hans-Rolf Schulten* \\ Fachhochschule Fresenius, Department of Trace Analysis, Dambachtal 20, \\ D-6200 Wiesbaden, Federal Republic of Germany. Received October 28, 1987
}

\begin{abstract}
The thermal degradation products formed from epoxy resins of the diglycidyl ether of bisphenol $A$ and the tetraglycidyl ether of tetrakis(hydroxyphenyl)ethane have been investigated by temperature-resolved in-source pyrolysis-field ionization mass spectrometry (Py-FIMS). Intense signals from thermal degradation products in the mass range $m / z 50-900$ were detected. Molecular ions containing up to three intact bisphenol A subunits were found as main degradation products, with rearrangement reactions being involved in the formation of abundant high-mass products. Most products contain at least one phenolic end group. The temperature resolution allowed distinction between released residual monomers and dimers, and the corresponding pyrolysates formed from thermal degradation of the polymer backbone. Furthermore, epoxy resins cured with bis(aminophenyl)methane, $m$-xylenediamine, and methylhexahydrophthalic acid anhydride (MeHHPA) were investigated. Degradation products containing both bisphenol A and bis(aminophenyl)methane as well as products containing bisphenol A units and MeHHPA were observed. On the other hand, products containing amino groups were not found for the resin cured with $m$-xylenediamine.
\end{abstract}

\section{Introduction}

Mass spectrometry (MS) is a powerful tool for the investigation of technical and biological polymers. ${ }^{1-4} \mathrm{~A}$ wide variety of pyrolysis methods and ionization modes are now available for investigation of their thermal degradation. ${ }^{1}$ Recently it has been demonstrated that pyrolysis (Py)GC/MS and direct pyrolysis-field ionization (Py-FI) MS give complementary analytical results, ${ }^{3}$ and the combination of these two methods has been successfully applied to the investigation of aromatic ${ }^{5}$ and aliphatic ${ }^{6}$ polyamides. Small molecules formed by thermal degradation are identified, after separation by coupled GC, with standard electron impact (EI) MS, whereas high-mass products that are unable to pass through the capillary column are observed by FIMS. FI is a soft ionization mode that is useful for the investigation of complex mixtures, since molecular ions are predominantly formed from the thermal degradation products, with fragmentation being largely avoided.

In the present work epoxy resins prepared from the diglycidyl ether of bisphenol A (DGEBA) and from the tetraglycidyl ether of tetrakis(hydroxyphenyl)ethane (TGETE) are investigated. In addition to the corresponding pure polymers, DGEBA-based resins cured with bis(aminophenyl)methane (DDM), $m$-xylenediamine, and methylhexahydrophthalic acid anhydride (MeHHPA) are used. One sample has been prepared by curing TGETE with DDM. These samples have recently been investigated by $\mathrm{Py}-\mathrm{GC} / \mathrm{MS}^{7}$ with a pyrolysis temperature of $600^{\circ} \mathrm{C}$. In these investigations, the thermal degradation products ethylene oxide, acetaldehyde, acrolein, acetone, allyl alcohol, and phenol were observed. The DGEBA-based resins formed bisphenol A, methylated DDMs, and MeHHPA, but products containing intact $m$-xylenedi-

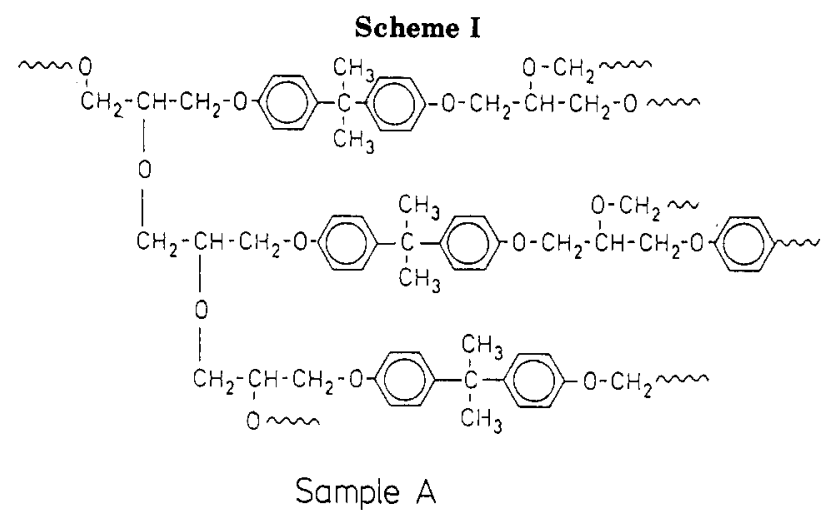

amine units were not observed.

The aim of our study was to monitor the presence of volatile oligomers remaining in the polymer after preparation and to investigate high-mass degradation products to obtain structural information. Indeed, products containing more than one bisphenol $\mathrm{A}$ unit or additional diamine units, which are not obtained by Py-GC/MS, are found in the FI spectra. The question is whether these high-mass signals can be used to give information about the linkage of the diphenol and diamine subunits.

\section{Experimental Section}

The Py-FIMS experiments were performed by using the modified ${ }^{8}$ direct-introduction system of a double-focusing Finnigan MAT 731 mass spectrometer. The samples were heated without any pretreatment from 50 to $750^{\circ} \mathrm{C}$ in high vacuum at about $10^{-3}$ $\mathrm{Pa}$ with a heating rate of $1.2^{\circ} \mathrm{C} \mathrm{s}^{-1}$. The mass range recorded was 50-900 daltons. The thermal degradation products were ionized at $8 \mathrm{kV}$ emitter potential, and the counterelectrode was at $-3 \mathrm{kV}$. All mass spectra were recorded electrically and averaged 